\title{
Dinámica de la germinación y agrotecnia para un eficiente desarrollo del botón de oro (Tithonia difersifolia)
}

\section{Dynamics of germination and agrotechnics for efficient development of}

\section{Tithonia diversifolia}

\author{
Sánchez Núñez Lina Marcela ${ }^{1}$; Hernández Bernal Diana Marcela ${ }^{1}$ y \\ Sánchez Moreno Hugo Vladimir ${ }^{2}$ \\ ${ }^{1} \mathrm{MVZ}$. Universidad de los Llanos y \\ ${ }^{2} \mathrm{MVZ}$. MSc Docente Universidad de los Llanos \\ vladimirsanchez@unillanos.edu.co
}

Recibido 11 de Abril 2014, Aceptado 12 de Septiembre 2014

\section{RESUMEN}

Se realizaron algunos trabajos con Tithonia diversifolia (botón de oro) y se compararon con los resultados reportados en la literatura en todo lo relacionado con su comportamiento agronómico en vivero, establecimiento en campo y sus diferentes usos. Los experimentos se basaron en la dinámica de germinación y la agrotecnia para un eficiente desarrollo del botón de oro. En las semanas 4, 6 y 8 las plantas presentaron un crecimiento inferior al $1 \%$, en comparación con las semanas 3, 5 y 7 ; se observó el papel benéfico funcional del incremento de las precipitaciones en el aumento exponencial del crecimiento de las plantas, así mismo, se observó un mayor crecimiento cuando el botón de oro se sembró con una estaca de $50 \mathrm{~cm}$, sus tallos brotaron más rápido, y el número de hojas y ramas siempre fue superior en comparación a las plantas que fueron sembradas con material vegetativo de $30 \mathrm{~cm}$, además soportaron mejor las épocas secas. Se concluye que en el piedemonte llanero la dinámica de germinación del botón de oro es más efectiva, si hay presencia de precipitaciones o disponibilidad de riego, como lo fue en este caso.

Palabras clave: Thitonia diversifolia, germinación, arbustiva forrajera. 


\begin{abstract}
A review of literature on Tithonia diversifolia (buttercup) plant is done, everything related to studies and research around it. Experimental work based on the dynamics of germination and agrotechnics for efficient development of buttercup explained. At weeks 4, 6 and 8 presented plant growth below 1\% compared to weeks 3, 5 and 7; the beneficial functional role of increased rainfall in the exponential rise in plant growth was observed, likewise, greater growth was observed when the buttercup was planted with a $50 \mathrm{~cm}$ stake, its stems sprouted faster, and the number of leaves and branches was always higher compared to the plants that were planted with vegetative material to $30 \mathrm{~cm}$, also withstood the dry seasons better. It is concluded that the germination dynamics of the buttercup is more effective, if there is the presence of rainfall or availability of irrigation, as it was in this case.
\end{abstract}

Keywords: Tithonia diversifolia, germination, forage shrub.

\title{
INTRODUCCIÓN
}

Tithonia diversifolia es una planta herbácea de la familia Asteracea, originaria de Centro América. Tiene un amplio rango de adaptación, tolera condiciones de acidez y baja fertilidad en el suelo, además tiene buena capacidad de producción de biomasa, rápido crecimiento y baja demanda de insumos y manejo para su cultivo. Presenta características nutricionales importantes para su consideración como especie con potencial en alimentación animal (Ríos, 1997).

En Colombia, se utiliza en apicultura y alimentación de vacas, conejos (Ríos, 1993), curíes, ovejas vacas, y cerdos. También se siembra como cerca viva para rodear sitios donde se ubican colmenas y áreas de bosque para protección de fuentes de agua (Ríos, 1997), también Se utiliza como especie ornamental y en parcelas de producción agrícola con alta diversidad para atraer insectos benéficos.

Desde hace un tiempo se ha venido hablando acerca de las bondades de la Tithonia diversifolia (botón de oro, árnica, margaritón, girasol mexicano, falso 
girasol) como fuente de suplementación de bajo costo para ganaderías del trópico bajo y medio (Soto et al., 2012), siendo además de fácil establecimiento en zonas como las del piedemonte llanero (Estrada, 2002).

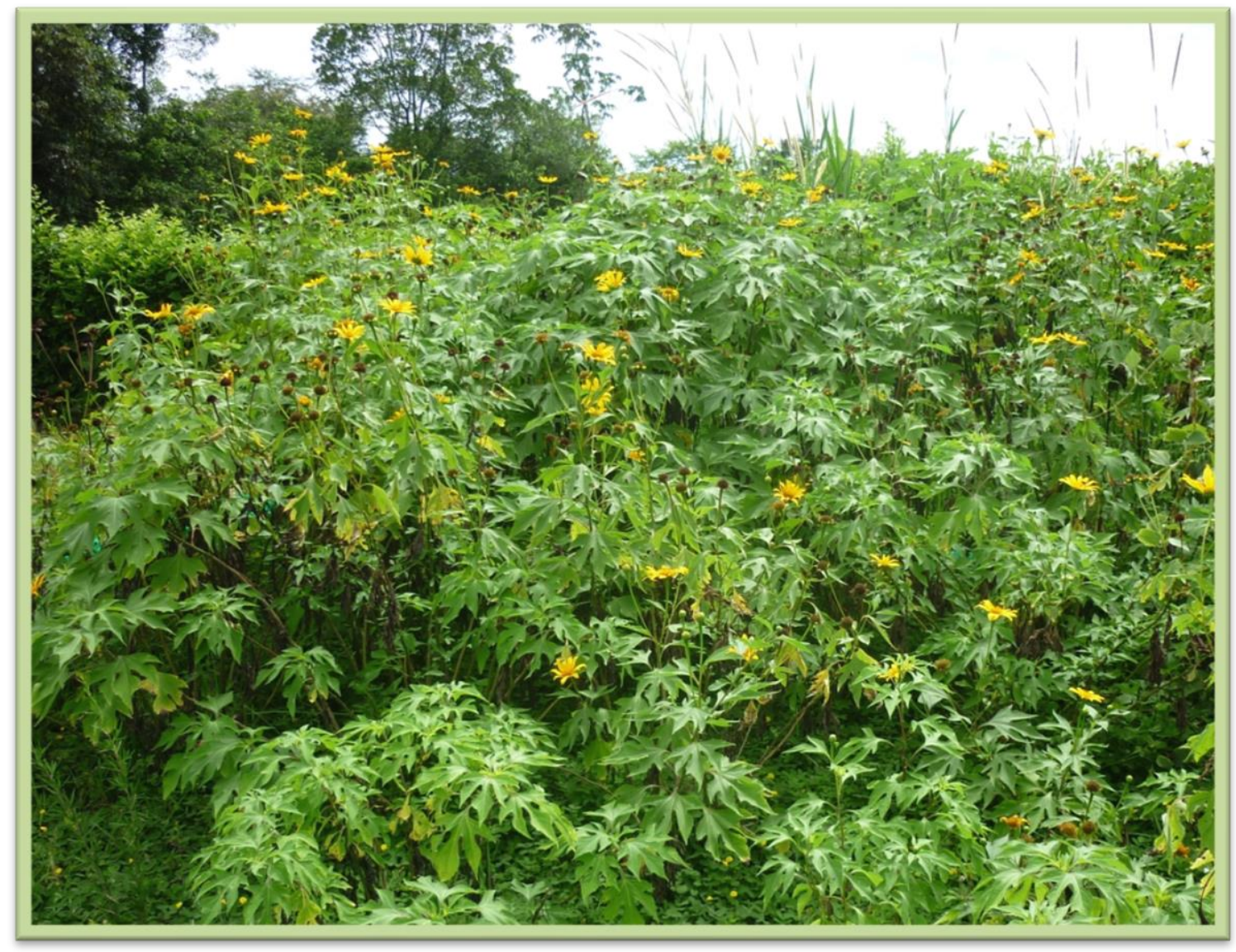

Fotografía 1. Tithonia diversifolia en países como México se le conoce al botón de oro como falso girasol por el color y forma de sus flores

A pesar de las ventajas para la alimentación animal, atracción de insectos, propiedades medicinales, cerca viva, abono verde y mejorador de suelos, la información acerca de la reproducción de botón de oro (Tithonia diversifolia) en condiciones de piedemonte llanero es mínima, limitando así su producción al método asexual o por estacas. Observaciones de campo han evidenciado que la propagación de $T$. diversifolia ocurre principalmente por vía asexual, mientras que la reproducción sexual es escasa o casi nula, esto sugiere problemas en alguna de las fases meióticas (Guerra et al., 2007). Es importante resaltar que el botón de oro es una planta rústica que no requiere muchos cuidados para su crecimiento y 
producción, lo cual facilita el manejo en plantación, creciendo bien en diferentes climas y sobre varias clases de suelos, tolerando condiciones de acidez y baja fertilidad, no obstante, se desarrolla mejor en clima medio con abundantes lluvias.

\section{Tithonia diversifolia COMO ESPECIE PROMISORIA PARA BANCOS FORRAJEROS}

T. diversifolia es una planta herbácea de 1.5 a $4.0 \mathrm{~m}$ de altura, con ramas fuertes subtomentosas, a menudo glabras, hojas alternas, pecioladas de 7 a $20 \mathrm{~cm}$ de largo y 4 a $20 \mathrm{~cm}$ de ancho, presenta 3 a 5 lóbulos profundos cuneados hasta subtruncados en la base, decurrentes en su mayoría en la base del pecíolo, bordes aserrados, pedúnculos de 4 a $20 \mathrm{~cm}$ de largo, lígulas amarillas a naranja de 3 a $6 \mathrm{~cm}$ y corolas amarillas de $8 \mathrm{~mm}$ de longitud (Guerra et al., 2007).

En China se realizó un estudio sobre sus dos nuevos compuestos $6-0-\beta-d-$ apiofuranosyl-trichocarpin y 1 -heptade-4,6-diino-3,10.16,17-tetraol-3-O- $\beta$ - $d$ glucopiranósido, y otros catorce compuestos conocidos que se obtuvieron de Tithonia diversifolia. Los nuevos compuestos pueden ser un marcador taxonómico que puede ser útil para diferenciarla de otras especies de Tithonia (Zhao et al., 2012). Lo anterior podría estar relacionado con la palatabilidad de accesiones que se dan en diferentes regiones en Colombia ya que productores de la zona norte del país reportan que esta especie no es consumida por los bovinos ante lo cual habría la necesidad de ampliar la investigación.

La inflorescencia se presenta en capítulos y está formada por pequeñas flores sésiles, dispuestas sobre un receptáculo convexo, provisto en su superficie de brácteas (páleas) rígidas, puntiagudas, de hasta $11 \mathrm{~mm}$ de largo (con algunos pelillos en su superficie), que abrazan las flores del disco, que está rodeado por

fuera por el involucro, que esta con acampanado ancho (hasta $4 \mathrm{~cm}$ ), constituido por numerosas brácteas (dispuestas en cuatro series), ovales y generalmente con el ápice redondeado, o bien las brácteas exteriores ovadas a redondeadas y con el ápice más o menos agudo, a veces cubiertas de pelillos (Pérez et al., 2009). 
Su hoja y tallo se han estudiado, en Brasil se investigaron caracteres microscópicos de esta planta medicinal, sus muestras de las hojas maduras y tallos jóvenes fueron seccionados y teñidos, también se realizaron pruebas histoquímicas y de microscopía electrónica de barrido. La hoja tiene estomas anomocíticos en ambos lados, mesófilo dorsiventral y varios haces vasculares colaterales organizados como un anillo en el nervio central, se observa un colénquima angular-tangencial, una endodermis evidente y tapas esclerenquimáticas adyacentes al floema (Duarte y Bonissoni, 2012).

La Tithonia diversifolia, es un arbusto que crece desde el nivel del mar hasta las tierras alto andinas, que tiene un enorme valor en sistemas ganaderos de todo tipo, es una planta herbácea muy ramificada que alcanza alturas hasta de cinco metros (Díaz y Murgueitio, 2008).

El bajo porcentaje de germinación (20\%) de los aquenios colectados en las en diferentes plantas de Tithonia diversifolia, puede ser atribuido al alto porcentaje de polen estéril $(65 \%)$ carente de núcleos espermáticos, lo que puede estar asociado a la baja reproducción sexual que presenta. Otras razones de la esterilidad del polen son las observadas en la división meiótica, donde se observan cromosomas rezagados en la anafase I, ocasionados por la falta de tensión sobre las fuerzas del huso, que ejercen las enzimas sensitivas del cinetocoro, evitando así el arrastre de los cromosomas hacia los polos, lo que puede desalinear un cromosoma, generando señales negativas que identifica la célula (Alcorcés et al., 2007).

La familia Asteracea posee unas 15.000 especies distribuidas por todo el mundo, el género Tithonia comprende diez especies originarias en Centro América, Tithonia diversifolia fue introducida a Filipinas, la India y Ceilán, también se registra en el Sur de México, Guatemala, Honduras, Salvador, Costa Rica, Panamá, Cuba, Venezuela y Colombia (Ríos, 1993; Chukwuka et al., 2007).

En Guatemala se registra su adaptación entre los 200 y 2300 msnm, en matorrales húmedos o secos. En Venezuela se encuentra en los estados de 
Carabobo, Aragua, Portuguesa y Trujillo entre los 300 y 1700 msnm (Ayeni et al., 1997). En Colombia esta planta crece en diferentes condiciones agroecológicas, desde el nivel del mar hasta 2700 metros en La Cocha, Nariño (Ríos, 1993), con precipitaciones que fluctúan entre 800 a $5000 \mathrm{~mm}$ y en distintos tipos de suelo, tolerando condiciones de acidez y baja fertilidad (Ríos, 1997). Se encuentra creciendo espontáneamente a orillas de caminos y ríos, observándose buen estado y producción desde el nivel del mar (cerca de Buenaventura, Valle), hasta los 2400 msnm en Rionegro, Antioquia, en suelos pobres y de mediana fertilidad.

La propagación de la especie se realiza principalmente con material vegetativo, porque no se ha tenido éxito de establecerla cuando se ha utilizado semilla, por falta de protocolos para obtener semillas viables, se ha utilizado material vegetativo proveniente de plantas jóvenes sin florecer, tomando tallos de aproximadamente $50 \mathrm{~cm}$ de longitud, 2.0 a $3.5 \mathrm{~cm}$ de diámetro y que posean de 4 o 5 yemas, estos son sembrados de manera horizontal o inclinada sin cubrirlos totalmente de tierra. La propagación por semilla obtenida en algunos cultivos, fue encontrada de forma natural en sitios donde existen plantas adultas, cuyas semillas caen al suelo en el momento propicio y encuentran las condiciones necesarias de humedad y luminosidad para germinar (Uribe et al., 2011).

En un ensayo en el cual se evaluó el número de raíces y porcentaje de prendimiento 15 días después de la siembra, de estacas procedentes de diferentes partes del tallo, se encontró un $94 \%$ de prendimiento en estacas tomadas de la parte más leñosa y $58 \%$ en las procedentes de la parte media. El número de raíces fue de 4.25 y 3.5 respectivamente (Salazar, 1992).

En un estudio sobre la caracterización agronómica durante la fase de establecimiento de seis especies con potencial forrajero: Tithonia diversifolia (Td), Cratylia argentea (Ca), Acalypha macrostachia (Am), Acalypha diversifolia (Ad), Gmelina arborea (Gm) y Moringa oleífera (Mo) en el piedemonte del Meta, Colombia, como resultados indicaron que a los 100 días, la sobrevivencia promedio fue de $82 \%$, siendo mayor para Td, Ca y Ga con valores de 100.100 y 
99\%, respectivamente, seguida de Ad con el 76\%, Am con 34\% y Mo con $0 \%$. El mayor vigor fue para Td y el menor para Am y Mo (Plazas y Sánchez, 2013).

\section{PRODUCCIÓN DE BIOMASA}

Se evaluó la producción de biomasa de Tithonia diversifolia en Buga (Colombia), a 1000 msnm con una precipitación bimodal de 1200 mm/año, en suelos de textura arcillosa y con pH de 6.5 (Ríos 1997; Hernández, 2008). El cultivo se estableció a partir de estacas tomadas del primero (parte más leñosa) y segundo tercio del tallo. Se aplicó riego después de la siembra y luego se colocó cobertura de bagazo de caña, hojarasca y pasto seco con el fin de conservar la humedad del suelo y evitar la competencia con otras especies de plantas, no se realizó ninguna labor de fertilización ni limpieza del cultivo y los riegos fueron escasos, tampoco se presentaron problemas fitosanitarios. Los tratamientos consistieron en tres densidades de siembra: 2.66, 1.77 y 1.33 plantas $/ \mathrm{m}^{2}$, evaluadas en dos alturas de corte sobre el nivel del suelo, el cultivo se encontraba en floración cuando se realizó el corte y contaba con 110 días de edad en ese momento (Ríos, 1997).

No se evidenció un efecto marcado de la densidad de siembra ni la altura de corte sobre la mayoría de las variables evaluadas, siendo mayor el número de tallos/planta en densidades de siembra menores, debido posiblemente a la disponibilidad de más espacio por planta, lo que permitió el desarrollo de una mayor cantidad de yemas, sin embargo este hecho no se vio reflejado en la producción de biomasa, debido a que los tallos eran más delgados. La planta parece guardar las proporciones entre sus diferentes partes: la relación tallo: hoja: flor de 5:3:2 se conservó en las tres densidades de siembra evaluadas y la producción potencial de biomasa en el primer corte fue de 2.66, 1.77 y 1.33 plantas $/ \mathrm{m}^{2}$, o de 82, 57 y 46 Ton/ha, respectivamente (Ríos, 1997; Ramírez, 2006).

Al someter el cultivo a cortes frecuentes se encontró una mayor producción de biomasa comestible por planta en las densidades menores 1.33 y 1.77 plantas $/ \mathrm{m}^{2}$ ), debido probablemente a la menor competencia por recursos. Sin 
embargo, si se establece la especie en monocultivo, es posible obtener mayor rendimiento por unidad de área en la densidad de 2.66 plantas/ha, aunque se podrían correr los riesgos fitosanitarios inherentes a esta forma de cultivo. La altura de corte o poda $(10$ y $50 \mathrm{~cm}$ ) solo afectó la altura de plantas en siete semanas con promedios de 135 vs 109 cm (Ríos, 1997).

La altura y frecuencia de corte o poda, y sus interacciones afectaron la producción de materia seca de $T$. diversifolia, mostrando una mayor altura y producción de biomasa cuando se dejan intervalos de tiempo de dos meses y se poda a $50 \mathrm{~cm}$ de altura, la producción de materia seca podría ser tan alta como 7.2 Ton/ha/año cantidad suficiente para mejorar la productividad del suelo en sistemas de transferencia de biomasa (Partey, 2011).

\section{CARACTERÍSTICAS NUTRICIONALES Y SU USO EN LA ALIMENTACIÓN ANIMAL}

Navarro y Rodríguez, (1990) realizaron análisis bromatológicos de T. diversifolia en cinco estados de desarrollo, después de un corte de uniformización a nivel del suelo: 1) crecimiento avanzado (30 días después del corte), 2) prefloración (50 días), 3 floración media (60 días), 4) floración completa (74 días) y 5) pasada la floración (89 días). Se tomaron muestras de hojas, peciolos, flores y tallos hasta $1.5 \mathrm{~cm}$ de diámetro, se encontraron diferencias para el porcentaje de proteína en los diferentes estados de desarrollo de la planta, puesto que a medida que avanzaba el desarrollo de la planta los datos de proteína disminuían, por lo tanto es importante tener en cuenta frecuencia de cortes para mantener la producción adecuada de biomasa comestibles, capacidad de recuperación sin detrimento del contenido de proteína, niveles superiores al $18 \%$.

Su contenido de proteína bruta varía desde $28.51 \%$ a los 30 días de edad hasta 14.84 de la materia seca, cuando se evaluó a los 89 días, se comprobó que la fibra cruda aumenta a través del tiempo, con valores de 1.63 y $3.83 \%$., variando su humedad de 85.9 (a los 30 días) hasta 76.75 (a los 89 días). Los contenidos de calcio y fosforo, expresados como porcentaje de la materia seca, disminuyeron a 
medida que se desarrollaba la planta, de $2.25 \%$ a $1.65 \%$ y de 0.39 a 0.32 respectivamente mientras que el magnesio aumentó de 0.046 y $0.069 \%$ (Guerra et al., 2007).

Una caracterización bromatológica de Tithonia diversifolia (Hemsl.) A. Gray en dos etapas de su ciclo fisiológico (30 y 60 días) fue realizada en Cuba, en los períodos lluvioso (PLL) y poco lluvioso (PPLL), donde se establecieron seis tratamientos derivados de la relación entre las fracciones comestibles (hojas, tallos tiernos y hojas más tallos tiernos) y los momentos del ciclo fisiológico de la planta. Para el PLL y el PPLL, el follaje de Tithonia presentó los mejores valores de proteína a los 30 días $(29.79 \%$ y $28.69 \%)$, Mg ( $0.094 \%$ y $0.210 \%$ ) y ceniza ( $16.32 \%$ y $20.59 \%)$ respectivamente, con resultados similares entre las fracciones comestibles para las dos etapas del ciclo fisiológico, en los dos períodos evaluados. El contenido de FB en los tallos tiernos a los 60 días fue el más representativo en ambos períodos (5.29 y $5.27 \%$, respectivamente). Integralmente, la fracción hoja más tallo tierno alcanzó los mejores valores nutricionales (Lezcano et al., 2012).

En Colombia se evaluó una dieta para ovinos de pelo con 50 y $100 \%$ de $T$. diversifolia fresca, bloque multinutricional (10\% de urea) a voluntad y follaje de matarratón (Gliricidia sepium 3\% del peso vivo en base fresca), complementando la dieta de $50 \%$ con cogollo de caña de azúcar, en la dieta de 50\% los animales consumieron $868 \mathrm{~g} /$ día en base fresca, lo que equivale a $369 \mathrm{~g} /$ día en base seca, en la de $100 \%$ el consumo fresco fue de $1668 \mathrm{~g} /$ día, correspondiente a $712 \mathrm{~g} / \mathrm{día}$ en base seca (Ríos, 1997).

Eslava et al., (2013) en los Llanos Orientales de Colombia, evaluaron la selectividad y palatabilidad de cuatro especies arbustivas con potencial en alimentación animal, establecidas en bancos forrajeros, se eligieron 12 animales homogéneos al azar, de $220 \mathrm{~kg}$ en promedio, los cuales tuvieron acceso de manera individual a forraje (60 días de edad) de Cratylia argentea, Leucaena leucocephala, Moringa oleifera y Tithonia diversifolia (2 kg por tratamiento). Se realizó análisis bromatológico a las cuatro arbustivas para evaluar proteína cruda (PC), digestibilidad in vitro de la materia seca (DIVMS), fibra detergente neutro 
(FDN) y fibra detergente acido (FDA). La especie que mayor consumo presentó fue C. argentea (754.1 g), seguida de L. leucocephala (678.3 g), T. diversifolia $(597.5 \mathrm{~g})$ y $M$. oleifera $(28.3 \mathrm{~g})$. Con respecto al tiempo de consumo, se presentaron diferencias significativas entre las cuatro especies $7.4 \pm 0.14 ; 6.0 \pm$ $0.14 ; 0.13 \pm 0.03$ y $4.5 \pm 0.13$ (C. argentea, L. leucocephala, M. oleifera y $T$. diversifolia, respectivamente).

En otro estudio, el consumo de alimento y digestibilidad fueron evaluados con cuatro ovinos en jaulas metabólicas alimentados con pasto Taiwán de baja calidad suplementados con Tithonia diversifolia en niveles: 20.35 y 50\%, se compararon con un control alimentado con concentrado de $20 \%$ de proteína en un diseño cuadrado latino (Ramírez et al., 2010). Así mismo Rosales, (1996) ha encontrado una alta degradabilidad de la materia seca, especialmente a las 48 horas $(33,50$. 83 y $90 \%$ a las $0.12,24$ y 48 horas respectivamente).

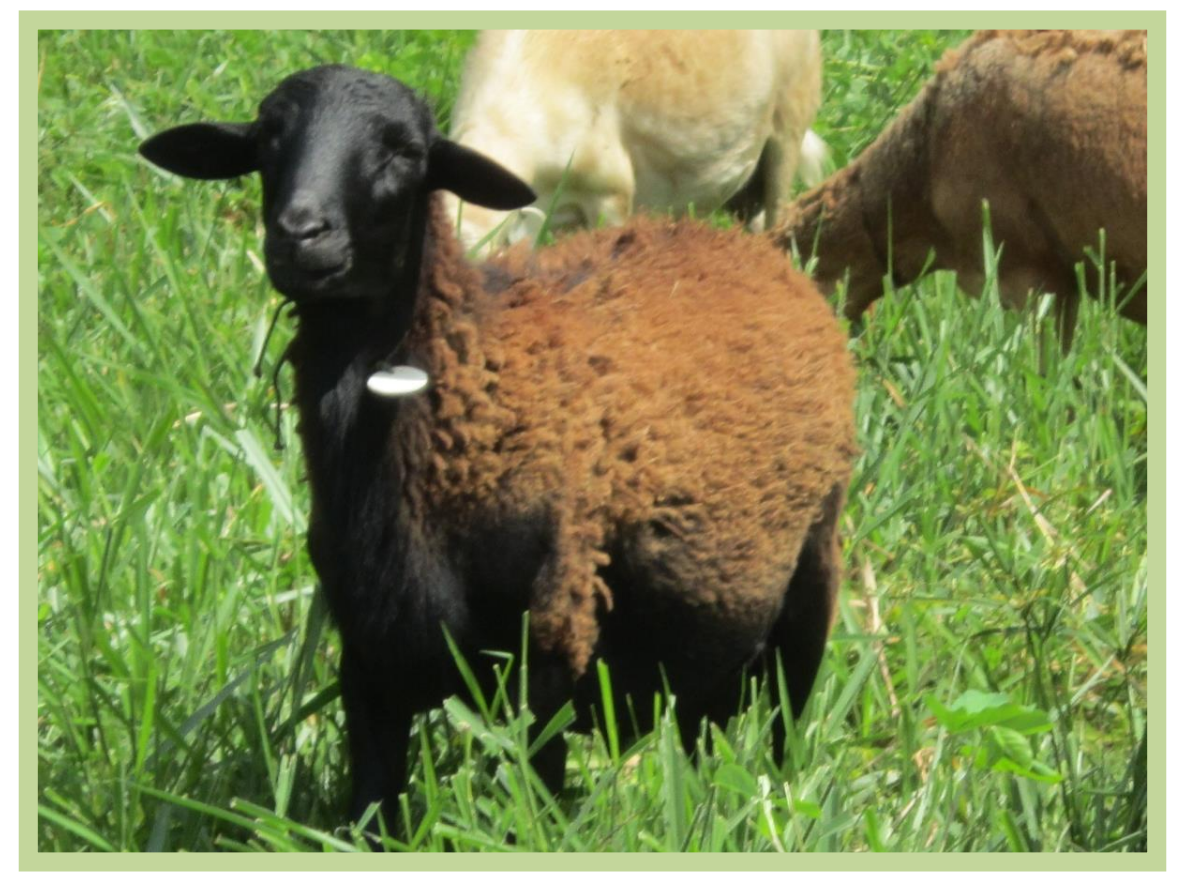

Fotografía 2. Se ha demostrado en la alimentación de ovinos que al utilizar botón de oro como componente de su dieta se eleva el consumo y por tanto su peso de los animales

Cairns, (1997) afirma que $T$. diversifolia es apreciada por los apicultores como fuente de néctar en Luzon, Filipinas y en la zona cafetera de Colombia, los 
apiarios se rodean con una franja ancha de $T$. diversifolia, sembrada a partir de estacas a $1 \mathrm{~m}$ de distancia, es importante determinar tres anillos de corte, los cuales se cosechan en forma escalonada con un intervalo de 4 meses entre ellos, estableciendo una frecuencia anual de corte en las plantas, de esta manera hay disponibilidad de flores todo el año para la alimentación de las abejas. Este cultivo cumple también con las funciones de rompevientos y protección del apiario, la biomasa producida por las plantas se deja en el sitio, para su descomposición e incorporación lenta al suelo. En Restrepo, Valle del Cauca, Colombia, existe un cultivo con diez años de edad, en buen estado, bajo este sistema de mínimo manejo y sin aplicación de agroquímicos (Ríos y Salazar, 1995).

En Colombia, se ha observado un excelente consumo de sus hojas por vacas Holstein en ramoneo a 2400 msnm (Ríos, 1997), campesinos de Dagua y El Dovio ofrecen para alimentación de vacas $T$. diversifolia picada en mezcla con otros forrajes como nacedero (Trichanthera gigantea), chachafruto (Erythrina edulis), morera (Morus alba) y cogollo de caña (Sacharum oficinarum) (Iglesias, 2003). Solarte, (1994) reporta que Tithonia se utiliza como parte de la dieta de cerdos en mezcla con otros forrajes como nacedero (Trichanthera gigantea), plátano (Musa spp.), cidra (Chayota edulis) y otros recursos locales, también se ha visto en fincas como componente de la dieta de conejos, curíes (Cavia porcellus) cerdos y vacas, suministrándola también a búfalos.

Como otra forma de alimentación, se evaluó la harina de botón de oro en dietas para 24 conejos Nueva Zelanda blancos destetos en etapa de crecimiento (35 días y $900 \mathrm{~g}$ de peso promedio) incluyendo tres niveles de harina de botón de oro ( $\mathrm{T} 1=15 \%, \mathrm{~T} 2=30 \%$ y $\mathrm{T} 3=45 \%$ ) y un testigo (T4) con concentrado comercial. Las dietas fueron isocalóricas $(2.400 \mathrm{Kcal})$ e isoproteicas $(16 \%)$. El consumo de materia seca fue similar $(\mathrm{P}<0.05)$, fueron mejor la ganancia de peso y conversión alimentaria $(\mathrm{P}<0.05)$ en comparación con el testigo $\left(27.3 \mathrm{~g}^{-\mathrm{d}^{-1}}\right.$ y 3,0 respectivamente), T1 y T2 emplearon menor número de días para alcanzar 2.000 g (44,3 y 52,1 días respectivamente), T2 presentó la mayor tasa de retorno marginal (Quintero et al., 2007). 
Es un recurso forrajero importante en producción animal, como ensilaje para alimentación de ovinos de carne en el Piedemonte del Meta en Colombia, debido a sus bondades nutricionales y de propagación, por lo tanto se evaluó la dinámica de fermentación y digestibilidad in vitro de ensilaje de Tithonia considerando cuatro períodos de maduración: UNO (botón sin ensilar), DOS (1, 3, 7 y 15 días), TRES (28, 42, 63 y 91 días) y CUATRO (119 días) (Roa et al., 2013). La degradación de la materia seca in vitro fue menor $(\mathrm{P}<0.05)$ en el periodo CUATRO. La digestibilidad de la fibra detergente ácido se incrementó $(P<0.05)$ con el proceso de ensilado, entre uno a 15 días, disminuyendo a los 119 días, quedando demostrado que la pared celular del botón de oro es aprovechada con mayor intensidad, cuando el tiempo de ensilaje está entre 1 a 92 días de conservación, decreciendo a los 119, cuando se disminuye la concentración de ácido láctico. Otro estudio, también en el piedemonte llanero de Colombia fue la evaluación agronómica a nivel de finca, de bancos forrajeros asociados con Tithonia diversifolia, Verbesina sp. Tourneforthia sp., Cratylia argentea, y Acalypha macrostachia, donde se obtuvo un promedio de producción de MS de 510.5 $\mathrm{kg} / \mathrm{MS} / \mathrm{Ha}$ con una relación hoja: tallo de 66.5: 33.5\% y una altura de planta promedio de $58.05 \mathrm{~cm}$, y un desplazamiento lateral promedio de $73.2 \mathrm{~cm}$, el corte se realizó a $20 \mathrm{~cm}$ del suelo (Plazas, 2010).

Ríos, (1997) afirma que se utiliza para alimentación de cabras en un sistema de corte y acarreo en Mindanao, Filipinas, aplicando el estiércol de los animales en los callejones del cultivo, se combina en este sistema los beneficios de la producción pecuaria, el reciclaje eficiente de nutrientes y la conservación de suelos. También se aprovecha para el ramoneo de ovejas y, en Luzón (Filipinas), algunos agricultores esparcen hojas de $T$. diversifolia en los estanques para ser consumida por tilapias. Adicionalmente en Indonesia se han realizado ensayos con resultados promisorios, al incorporar hojas de esta especie en raciones para alimentación de gallinas.

Un sistema de producción en Venezuela utiliza Tithonia como forraje fresco sin picar, este se ofrece colgado para el consumo de ovejas y cabras, como parte de 
una dieta con cogollo de caña y pasto elefante, en la tarde se ofrece a los animales, nacedero (Trichanthera gigantea), matarratón (Gliricidia sepium) y cañafístola (Cassia moschata) (Savón, 2006). En Nigeria, se utilizaron 24 cerdos machos para determinar el efecto del ensilaje de Tithonia diversifolia secada al sol. Se evaluó su desempeño en el crecimiento y la utilización de nitrógeno en la inclusión en la dieta de 10. 20 y 30\%, considerando como recurso novedoso el valor potencial de Tithonia diversifolia secada al sol con niveles de hasta un $10 \%$ de proteína cruda siendo compatibles para un rendimiento, crecimiento óptimo y la utilización de nitrógeno (Pedroso, 2008).

Respecto a su uso en aplicaciones agroindustriales, se realizó una investigación en Colombia sobre sus potencialidades para la conservación postcosecha: un análisis de calidad de 44 introducciones utilizando la colección de $T$. diversifolia de la Universidad Nacional de Colombia (sede Palmira) con una edad de 60 días, donde se evaluó materia seca, peso de la planta, peso de las hojas, peso de los tallos frescos (preciolos) y altura, con el fin de estimar relación hoja tallo y producción de biuomasa comestible. Los resultados de las 10 mejores introducciones indican una altura promedio de las plantas de $2.61 \pm 0.62 \mathrm{~m}$, MS promedio de $16.8 \%$; relación hoja: tallo de $1.81 \pm 0.49$, con una producción anual de $74 \mathrm{Ton} / \mathrm{ha}$. Se puede concluir que las introducciones poseen las características adecuadas para producir un alto rendimiento de forraje por hectárea para ser usado como alimento en fresco (Velasco et al., 2013).

En pruebas biológicas de crecimiento de pollitos a partir de siete días de edad, alimentados durante siete días con una dieta en la cual se sustituyó el $20 \%$ de concentrado comercial por follaje seco y molido de $T$. diversifolia, se obtuvo un alto consumo y ganancia de peso, de igual manera, la conversión fue eficiente, entre 25 a 50\% frente al testigo. Estos resultados se explican por el buen contenido de proteína de la especie, su alta digestibilidad de la materia seca y bajo contenido de fenoles y saponinas (Vargas, 1996). 


\section{CERCA VIVA Y ROMPE VIENTOS}

En Colombia (Rio Frío, Valle del Cauca) en el aislamiento de fragmentos de bosque que cumplen funciones de protección y conservación de fuentes de agua, se estableció $T$. diversifolia como cerca viva (Ríos, 1997), en reemplazo de cercas con alambre de púas. En fincas de la zona de ladera del Valle del Cauca, se siembra $T$. diversifolia, asociada con otras especies forrajeras como Trichanthera gigantea, sembrando franjas de cada especie, también alrededor de parcelas de policultivo o en las cercas, se cosecha antes de floración para alimentar animales y se fertiliza con estiércol fresco de bovino o con lombricompuesto, también se siembra como rompevientos alrededor de apiarios en la zona cafetera colombiana.

\section{ABONO VERDE Y MEJORADOR DE SUELOS}

En Luzón (Filipinas), algunos agricultores consideran las parcelas con $T$. diversifolia como bancos de fertilizante. En la provincia de Mountain (Filipinas), es cosechada e incorporada como abono verde en campos de cultivo de arroz con inundación, debido a su rápido crecimiento, eficiente depuración de nutrientes del suelo, abundante producción de hojas y rápida descomposición, esta especie parece acelerar el reciclaje de nutrientes y permite la rehabilitación del suelo en un período corto de barbecho (Ríos, 1997).

En Costa Rica, al evaluar especies identificadas por agricultores entre ellas $T$. diversifolia, como favorables para la producción de fríjol bajo el sistema tapado, se encontró en sus hojas altos contenidos de fósforo, calcio y potasio (más de 2500 ppm). Al comparar la producción de fríjol en barbechos mejorados con diferentes especies se obtuvieron los siguientes resultados: en barbecho natural, $628 \mathrm{~kg} / \mathrm{ha}$; en barbecho con $T$. diversifolia, $749 \mathrm{~kg} / \mathrm{ha}$ y mayor producción de biomasa y fósforo (Ríos, 1997).

T. diversifolia ayuda a la depuración de nutrientes lábiles del suelo que se pierden por lixiviación, en el caso del fósforo, la asociación con micorrizas puede estar cumpliendo un rol importante en su movilización. En la provincia de Bukidnon, Filipinas, $T$. diversifolia es utilizada para recuperar y mejorar áreas invadidas por el 
pasto Imperata cylindrica, la sombra de T. diversifolia controla el pasto en un año, al final del segundo año, se cortan las plantas de Tithonia y se siembra un nuevo cultivo sin necesidad de aplicar fertilizantes ni arar, porque se mejoran las propiedades físicas del suelo, es así como se puede cambiar el concepto de barbechos con malezas al de abono verde o cultivo de cobertura (Cairns, 1997).

En Nigeria, se estudiaron las propiedades de coberturas de Chromolaena y Tithonia evaluando: rendimiento, composición y crecimiento del tubérculo de yam blanco (Dioscorea rotundat), se observó que los abonos de Chromolaena y Tithonia reducen la densidad aparente del suelo y la temperatura, también se incrementaron las concentraciones de materia orgánica, nitrógeno $(N)$, fosforo $(P)$, potasio $(\mathrm{K})$, calcio (Ca) y magnesio $(\mathrm{Mg})$ en el suelo, y $\mathrm{N}, \mathrm{P}, \mathrm{K}, \mathrm{Ca}$ y $\mathrm{Mg}$ en las hojas (Agbede et al., 2013).

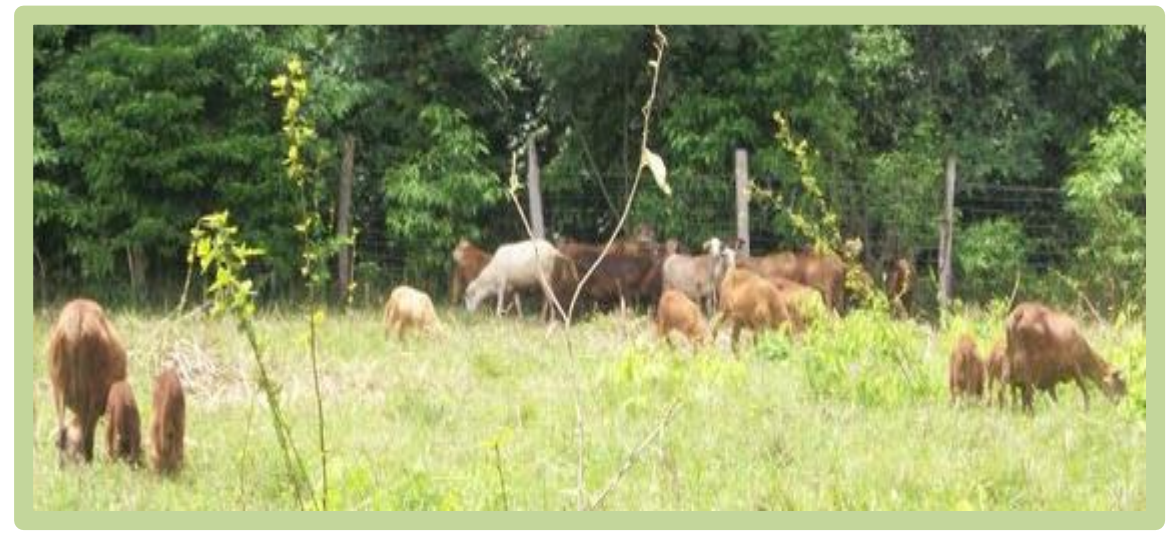

Fotografía 3. El botón de oro es una buena alternativa para la alimentación de ganado en pastoreo con gramíneas de baja calidad nutricional

Se compararon la descomposición y los patrones de liberación de nutrientes de $T$. diversifolia con Senna spectabilis, Gliricidia sepium, Leucaena leucocephala y auriculiformis, que se utilizan comúnmente en los sistemas de transferencia de biomasa. Los resultados del estudio confirmaron altas concentraciones de $\mathrm{N}, \mathrm{P}, \mathrm{K}$ en $T$. diversifolia comparables con los niveles registrados en las cuatro especies de leguminosas, además Tithonia registró el mayor porcentaje de descomposición y tasa de liberación de nutrientes $(P<0.05)$. Era evidente a partir del estudio, que la descomposición y la velocidad de liberación de nutrientes de estas especies, están 
relacionadas con la calidad del material de la hoja, siendo el $\mathrm{P}$ y $\mathrm{Mg}$ los más influyentes para estos procesos de liberación de nutrientes. Por esta razón, sería imprescindible tener en cuenta las concentraciones de $\mathrm{P}$ y $\mathrm{Mg}$ en la selección de materiales de plantas para abono verde (Partey et al., 2011).

Los metabolitos secundarios como taninos, oxalatos, glucosinolatos, saponinas, alcaloides, fenoles y demás, provenientes de forrajes como: sauco, botón de oro y morera, son una alternativa para reducir las emisiones de metano entérico en los rumiantes, estos metabolitos secundarios se extraen usando dos solventes (agua y etanol 95/100 mL), seguido de la identificación de los componentes de los dos tipos de extractos, acuoso (EAC) y alcohólico (EAL) (Vásquez et al., 2013).

En Colombia, se aplicaron técnicas analíticas e instrumentales de la AOAC, (2006) para detectar el efecto de las condiciones de conductividad eléctrica a muestras de dos hojas con peciolo de Gliricidia sepium y Tithonia diversifolia, en diferentes condiciones edáficas (departamentos del Valle del Cauca y Cesar), para determinar contenido de metabolitos que se producen en sus células como: polifenoles, taninos totales, taninos condensados y saponinas. Los resultados obtenidos para cada variable se sometieron a un análisis de varianza en doble vía, Se concluyó que el contenido de polifenoles, taninos totales, taninos condensados y saponinas fueron similares para las tres zonas muestreadas presentándose una alta correlación entre el valor de la conductividad eléctrica del suelo y el contenido de metabolitos secundarios (Santacoloma y Granados, 2012).

Estudios más recientes como el que se llevó a cabo en Brasil donde se ha utilizado Tithonia diversifolia en la medicina popular para el tratamiento de abscesos, infecciones microbiológicas, picaduras de serpientes, la malaria y la diabetes. Ambas propiedades anti-inflamatorias y anti-malaria han sido identificadas utilizando ensayos apropiados, pero las dosis eficaces han demostrado efectos tóxicos para los animales de experimentación, la mayor parte de las actividades farmacológicas se han atribuido a lactonas sesquiterpénicas (STL) y algunos derivados del ácido clorogénico (AC) en las hojas de esta especie. Se evaluó la toxicidad de dosis repetidas de un extracto acuoso (AE) de 
Tithonia diversifolia de las hojas comparándolo con un extracto rico en STL (LRE) y un extracto polar (PE) sin STL pero rico en AC. El AE causó alteraciones en los parámetros hematológicos, pero pocas alteraciones en los parámetros bioquímicos y era relativamente segura en dosis inferiores a $100 \mathrm{mg} / \mathrm{kg}$. Sin embargo, el PE y LRE demostraron varios efectos adversos al dañar el hígado y los riñones, respectivamente (Donaire et al., 2013).

T. diversifolia tiene compuestos tóxicos en sus hojas como lo afirman Elufioye et al., (2009) quienes investigaron la toxicidad de un extracto etanólico de las hojas, que en Nigeria son usados para tratar la malaria, se demostró una relación dosisefecto tóxica lo cual dependía del tiempo, siendo reversible en el riñón e hígado, no se observó alteraciones en la morfología del corazón, el bazo y el cerebro. Se ha demostrado que el extracto de etanol al $70 \%$ de las hojas de Tithonia diversifolia, reducen la parasitemia en ratones infectados con Plasmodium, sin embargo, se observó daños en el riñón e hígado a la dosis más baja en que fueron sometidos los animales.

Fueron encontradas mayores concentraciones de oxalato libre y aluminio (Al) en las raíces, en comparación con las hojas de Tithonia diversifolia en suelos de cultivo y no alterado (Reserva Ecológica en Venezuela), ambos tipos de suelo mostraron concentraciones similares de calcio $(\mathrm{Ca})$, manganeso $(\mathrm{Mn})$ y níquel $(\mathrm{Ni})$, mientras que las concentraciones de $\mathrm{Al}$, hierro $(\mathrm{Fe})$, cobre $(\mathrm{Cu})$ y zinc $(\mathrm{Zn})$ del campo de cultivo eran más altos que los del suelo no alterado. Se encontró en las plantas de suelos no alterados un mayor valor de oxalato libre concluyendo que el oxalato está actuando en $T$. diversifolia como quelante del $\mathrm{Al}$, evitando de este modo el efecto tóxico de este metal (Otusanya, et al., 2007).

Existe la posibilidad de remediar los suelos contaminados por el sector industrial a través del uso de ciertas plantas. En Ibadan (Nigeria), se investigó la capacidad de remediar con Helianthus annuus y Tithonia diversifolia el suelo contaminado con efluentes de la industria de la pintura, comparándolos con un tratamiento control, con dos niveles de fetilidad: minerales (MF); fertilizantes minerales orgánicos (OMF); se plantaron semillas Helianthus annuus a una distancia de $60 \times 30 \mathrm{~cm}^{2}$, 
siendo el porcentaje de eliminación de metales pesados con MF y la OMF así: Cu 32.5 y $41.6,30.3$ y $42.8 \mathrm{~Pb}$ y $\mathrm{Cd} 44.5$ y 56.7 , respectivamente con Tithonia diversifolia, del mismo modo, eliminó: cobre $(\mathrm{Cu}) 16.9$ y 23.4, 36.9 y 43.7 plomo $(\mathrm{Pb})$ y cadmio $(\mathrm{Cd}) 20.1$ y 35.1 respectivamente, se demostró que los testigos, donde no se aplicó fertilizante presentaron los porcentajes más bajos de eliminación, de acuerdo a los resultados se recomienda el uso de plantas de girasol y botón de oro, junto con la aplicación de OMF para remediar efectivamente los suelos contaminados con metales pesados, en particular en clima tropical (Jemutai et al., 2012).

\section{EXPERIENCIAS PERSONALES}

En base a sus virtudes reportadas en diferentes estudios, como la facilidad de propagación, resistencia, adaptabilidad en los diferentes ecosistemas, ventajas como suplemento alimenticio animal, usos en el campo medicinal, y como planta conservadora y recuperadora de la calidad del suelo, entre otros; se decidió desarrollar un estudio fundamentado en la revisión literaria sobre Tithonia diversifolia, el cual se llevó a cabo en la Finca Iriqué, en la vereda Los Andes, ubicada en Granada (Meta, Colombia), con una altitud de 372 msnm., temperatura promedio de $25.6-28^{\circ} \mathrm{C}$, precipitación promedio anual entre 1830 a $2800 \mathrm{~mm}$ y humedad relativa del $80 \%$.

En un terreno de $360 \mathrm{~m}^{2}$ se procedió a realizar la siembra asexual de Tithonia diversifolia a partir de estacas tomadas de otro cultivo que ya tenía 24 meses de establecido, ubicado en la Universidad de los Llanos, no se aplicó ningún tipo de fertilizante utilizando dos (2) alturas de estaca (30 y $50 \mathrm{~cm}$ ), se utilizó un diseño experimental en bloques al azar con dos repeticiones por tratamiento.

Se inició con la toma de datos semanales que incluyó: número de ramas, número de hojas por tallo, altura de las plantas, porcentaje de supervivencia. En las Gráficas 1 y 2, en las semanas 4, 6 y 8 se presentó un menor crecimiento de las plantas en comparación con las semanas 3,5 y 7 , se puede observar el papel benéfico funcional del incremento de las precipitaciones en el aumento 
exponencial del crecimiento de las plantas, teniendo en cuenta que el agua en fundamental en procesos vitales de la planta, como por ejemplo la fotosíntesis.
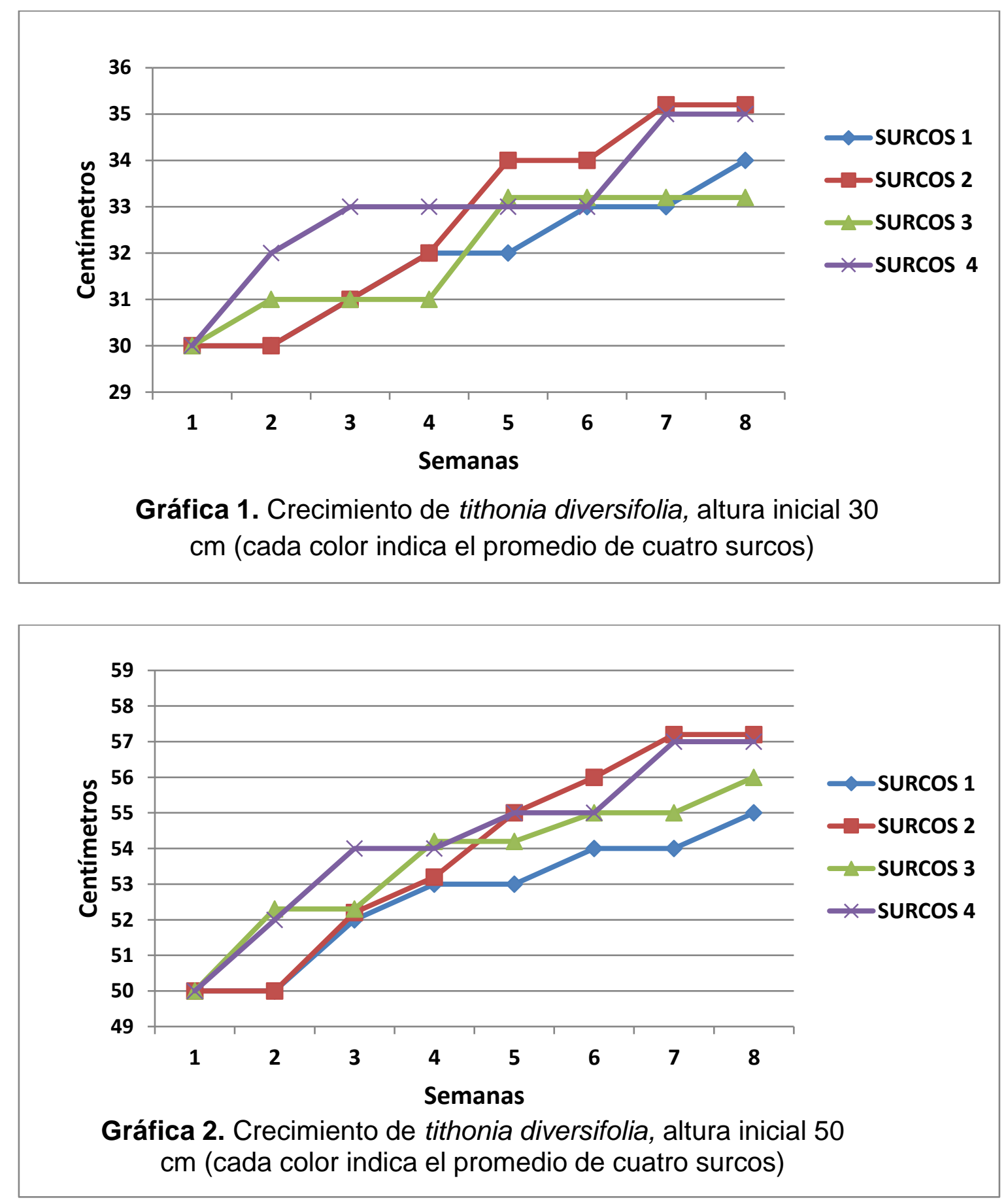

Así mismo, se observó un mayor crecimiento cuando el botón de oro se sembró con una estaca de $50 \mathrm{~cm}$, sus tallos brotaron más rápido, y el número de hojas y ramas, siempre fue superior en comparación a las plantas que fueron sembradas 
con material vegetativo de $30 \mathrm{~cm}$, lo cual se evidencio posteriormente puesto que las plantas $(50 \mathrm{~cm}$ de estaca) soportaron mejor las épocas secas.

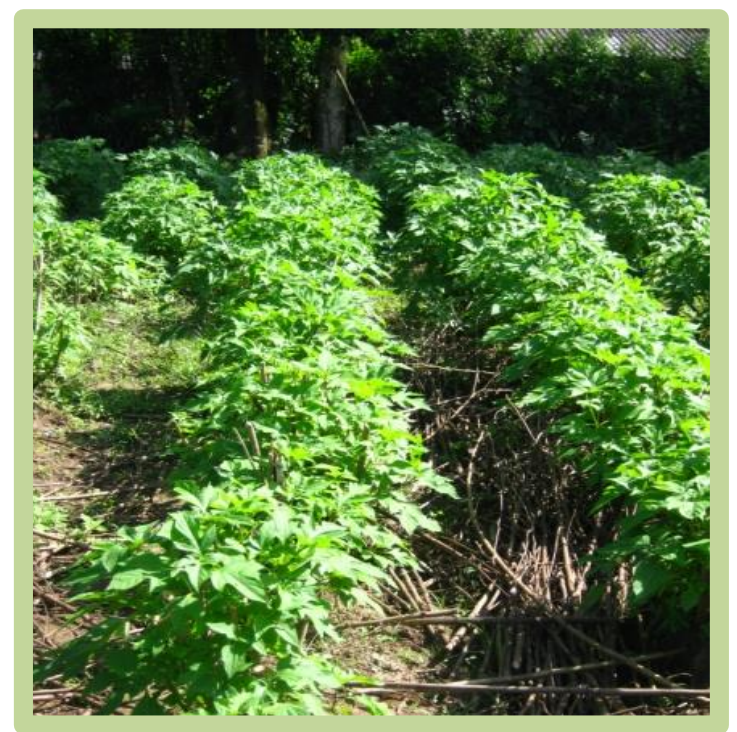

Fotografía 4. Se observó mayor crecimiento cuando el botón de oro se sembró con estacas de $50 \mathrm{~cm}$.

\section{CONCLUSIONES}

Tithonia diversifolia es una planta promisoria por sus múltiples usos, facilidad de propagación y adaptación a varios de los ecosistemas con los que cuenta la geografía Colombiana.

Existe una deficiencia en registros literarios sobre la propagación mediante material vegetativo, factor que incrementa el margen de error (método ensayoerror), aumentando la necesidad de material para la implementación con semillas sea más alto y menos productivo, por lo cual la propagación de esta planta se realiza casi en un $100 \%$ mediante el uso de estacas, que son fáciles de adquirir de otros cultivos, pero cabe resaltar que este tipo de propagación presenta algunas dificultades como el transporte y almacenamiento que solo se puede realizar durante periodos cortos, sin afectar la calidad de las estacas.

En algunas zonas de Colombia cuando la flor de la planta ya se encuentra seca la cortan y la colocan en la tierra, tapándola superficialmente hasta que germinan las 
primeras plántulas, se retiran los restos de la flor, siendo una de las pocas evidencias con las que se cuenta de reproducción con semilla.

Las plantas sembradas de $50 \mathrm{~cm}$ de altura y de un grosor de $2.5 \mathrm{~cm}$, crecen más rápido y soportan mejor las épocas secas.

\section{BIBLIOGRAFÍA}

1. Agbede, T., Adekiya, O., Ogeh, J. Effects of Chromolaena and Tithonia Mulches on Soil Properties, Leaf Nutrient Composition, Growth and Yam yield. West African Journal of Applied Ecology, 21 (1): 15-29. 2013.

2. Alcorcés N., Lárez A., Mayz, J. Adiciones al conocimiento citogenético de Tithonia diversifolia (hemsl.) A. gray (Asteraceae). Acta botánica Venezuelica, 30 (2): 267-275. 2007.

3. Association of Official Analytical Chemists (AOAC). Official Methods of Analysis. $18^{\text {th }}$ Ed., Washington, D.C. 2006.

4. Ayeni A., Lordbanjou D., Majek B. Tithonia diversifolia (Mexican sunflower) in southwestern Nigeria: occurrence and growth habit. Weed Research, 37 (6): 443-449. 1997.

5. Cairns M. A Property Rights Dimension of Indigenous Fallow Management (IFM). Asia-Pacific Resource Tenure Network, (ARTN) Indonesia. 1997.

6. Chukwuka K., Ogunyemi S., Fawole I. Ecological distribution of Tithonia diversifolia (Hemsl). A. Gray-A New exotic weed in Nigeria. Journal of Biological Sciences, 7 (5): 709-719. 2007.

7. Díaz Z., Murgueitio E. El botón de oro: arbusto de gran utilidad para sistemas ganaderos de tierra caliente y de montaña. Carta Fedegan N. 108, p 54-63. 2008.

8. Donaire F., Oliveira R., Aparecida D., Gobbo-Neto L., Batista Da Costa, F. Repeateddose toxicological studies of Tithonia diversifolia (Hemsl.) A. gray and identification of the toxic compounds. Journal of Ethnopharmacology, 147 (2): 389-394. 2013.

9. Duarte M., Bonissoni C. Leaf and stem microscopic identification of Tithonia diversifolia (Hemsl.) A. Gray (Asteraceae). Brazilian Journal of Pharmaceutical Sciences, 48 (1): 109-116. 2012.

10. Elufioye A., Alatise O., Fakoya F., Agbedahunsi J., Houghton P. Toxicity studies of Tithonia diversifolia A. Gray (Asteraceae) in rats. Journal of Ethnopharmacology, 122 (2) 410-415. 2009.

11. Eslava A., Gómez L., Navas A., Calvache I., y Ardila A. Evaluación de la selectividad y palatabilidad de especies arbustivas forrajeras por bovinos en los llanos orientales de Colombia. En: IV Seminario Internacional de Agroforestería. Quibdó, Chocó, Colombia, 2013.

12. Estrada, J. Pastos y forrajes para el trópico colombiano. Manizales: Ed Universidad de Caldas. 511 p. 2002.

13. Guerra N., Lárez A., Mayz J. Adiciones al conocimiento citogenético de Tithonia diversifolia (HEMSL.) A. gray (Asteraceae). Acta Botánica Venezuelica, 30 (2): 267275. 2007.

14. Hernández, A. Factores agronómicos que influyen en la producción de Tithonia diversifolia en la provincia de Matanzas. Trabajo de Curso. EEPF Indio Hatuey. Sede Universitaria de Perico. Matanzas, Cuba. 2008. 
15. Iglesias J. Los sistemas silvopastoriles, una alternativa para la crianza de bovinos jóvenes en condiciones de bajos insumos. La Habana, Cuba. Tesis en opción al grado de Doctor en Ciencias Veterinarias. ICA, 110 p. 2003.

16. Jemutai K., Orata F., Getenga Z. The influence of filter mud compost and Tithonia diversifolia leaves on the dissipation of diuron in soils within the nzoia river drainage basin, Kenya. Bulletin of Environmental Contamination and Toxicology, 89 (2): 328333. 2012.

17. Lezcano Y., Soca M., Ojeda F., Roque E., Fontes D., Montejo I., Santana H., Martínez J., Cubillas N. Caracterización bromatológica de Tithonia diversifolia (Hemsl.) A. Gray en dos etapas de su ciclo fisiológico. Pastos y Forrajes, 35 (3): 275-282. 2012.

18. Navarro F., Rodríguez E. Estudio de algunos aspectos bromatológicos del Mirasol (Tithonia diversifolia Hemsl y Gray) como posible alternativa de alimentación animal. Tesis Universidad del Tolima. Ibagué, Tolima. Colombia. 86 p. 1990.

19. Otusanya O., Ilori O., Adelusi A. Allelopathic effects of Tithonia diversifolia (Hemsl) A. Gray on germination and growth of Amaranthus cruentus. Research Journal of Environmental Sciences, 1 (6): 285-293. 2007.

20. Partey S., Quashie S., Thevathasan N., Gordon A. Decomposition and nutrient release patterns of the leaf biomass of the wild sunflower (Tithonia diversifolia): a comparative study with four leguminous agroforestry species. Agroforestry Systems, 81 (2): 123-134. 2011.

21. Pedroso A. Empleo de la Tithonia en la preceba de cerdos en la EEPF "Indio Hatuey": Trabajo de Curso. EEPF "Indio Hatuey"- Sede Universitaria de Perico. Matanzas, Cuba. 38 p. 2008.

22. Pérez A., Montejo I., Iglesias J., López O., Martín G., García D., Idolkis M., Hernández A. Tithonia diversifolia (Hemsl.) A. Gray. Pastos y Forrajes, 32 (1): 1-15. 2009.

23. Plazas $\mathrm{C}$. Evaluación agronómica a nivel de finca, de bancos forrajeros asociados con Tithonia diversifolia, Verbesina sp. Tourneforthia sp., Cratylia argentea, y Acalypha macrostachia. Experiencias con pequeños productores del Piedemonte del Meta. Revista Electrónica Sistemas de Producción Agroecológicos, 1 (1): 74-94. 2010.

24. Plazas C., Sánchez V. Caracterización agronómica durante la fase de establecimiento de seis especies con potencial forrajero en el piedemonte del Meta, Colombia. Memorias IV Seminario Internacional de Agroforestería. Quibdó, Chocó, Colombia, 2013.

25. Quintero V., García G., Peláez A. Evaluación de harina de botón de oro en dietas para conejos en etapa de crecimiento. Acta Agronómica (Palmira), 56 (4): 203-206. 2007.

26. Ramírez U. Productividad agronómica del arbusto forrajero Tithonia diversifolia en Yucatán, México. Matanzas, Cuba. En: IV Congreso Latinoamericano de Agroforestería para la producción animal sostenible y III Simposio sobre sistemas silvopastoriles para la producción ganadera sostenible. EEPF "Indio Hatuey", Matanzas, Cuba. 35 p. 2006.

27. Ramírez U., Sanginés J, Escobedo J., Cen F., Rivera J., Lara P. Effect of diet inclusion of Tithonia diversifolia on feed intake, digestibility and nitrogen balance in tropical sheep. Agroforestry Systems, 80 (2): 295-302. 2010.

28. Ríos C. Efecto de la densidad de siembra y altura de corte sobre la producción de biomasa del botón de oro Tithonia diversifolia (Hemsl) Gray, evaluada en cortes sucesivos. Investigación, validación y capacitación en Sistemas Agropecuarios Sostenibles. Convenio CETEC-IMCA-CIPAV. Informe de avance. Cali. 81 p. 1993. 
29. Ríos C. Tithonia diversifolia (hemsl.) Gray, una planta con potencial para la producción sostenible en el trópico. Agroforestería para la Producción Animal en Latinoamérica, p 217-230. 1997.

30. Ríos C., Salazar A. Botón de oro (Tithonia diversifolia (Hemsl.) Gray) una fuente proteica alternativa para el trópico. Livestock Research for Rural Development, 6 (3): p 75-87. 1995.

31. Roa M., Céspedes D., Lozada C. P. Maduración del ensilaje de botón de oro (Tithonia diversifolia) en diferentes periodos y su efecto en la digestibilidad in vitro e in vivo en ovinos de carne. Revista Colombiana de Ciencias Pecuarias, 26 (Suplemento): p 454. 2013.

32. Rosales M. In vitro assessment of the nutritive value of mixtures of leaves from tropical fodder trees. Tesis de Doctorado D.Phil. Departament of Plant Sciences, Oxford University. Oxford, UK. p. 214. 1996.

33. Salazar A. Evaluación agronómica del botón de oro (Tithonia diversifolia - familia compuesta) y el pinocho (Malvaviscus penduliflorus - familia malvaceae). Informe de becarios de la Fundación Centro para la Investigación en Sistemas Sostenibles de Producción Agropecuaria, Cali. p 27-31. 1992.

34. Santacoloma L., Granados J. Interrelación entre el contenido de metabolitos secundarios de las especies Gliricidia sepium y Tithonia diversifolia y algunas propiedades físicoquímicas del suelo. Revista de Investigación Agraria y Ambiental, 3 (1): 53-62. 2012.

35. Savón L. Alimentación no convencional de especies monogástricas: utilización de alimentos altos en fibras. Conferencia Magistral. Instituto de Ciencia Animal. La Habana, Cuba. 2006.

36. Solarte A. Experiencias de investigación participativa en sistemas de Producción Animal en dos zonas del Valle del Cauca. En: III Seminario Internacional Desarrollo Sostenible de Sistemas Agrarios, p 49-72. 1994.

37. Soto M., Molina F., González I., González J., Sánchez E. Efecto de la altura y frecuencia de corte sobre la producción de materia. Zootecnia Tropical, 30 (4): 315325. 2012.

38. Uribe F., Zuluaga A., Murgueitio E., Valencia L., Zapata A., Solarte L., et al. Establecimiento y manejo de sistemas silvopastoriles. Proyecto ganadería colombiana sostenible. 78 p. 2011.

39. Vargas J. Caracterización de recursos forrajeros disponibles en tres agroecosistemas del Valle del Cauca. Tesis Maestría en Desarrollo Sostenible de Sistemas Agrarios. Universidad Javeriana - IMCA - CIPAV. p 135-152. 1996.

40. Vásquez D., Rodríguez T., Mestra L., Mancipe E., Ariza C., Mayorga O. Extracción e identificación de metabolitos secundarios de sauco (Sambucus nigra), botón de oro (Tithonia diversifolia) y morera (Morus alba), como potenciales inhibidores de la metanogénesis ruminal bajo condiciones anaeróbicas in vitro. Revista Colombiana de Ciencias Pecuaria, 26 (Suplemento), p 450. 2013.

41. Velasco A., Vilma H., Ortiz S. Potencialidades de Tithonia diversifolia para la conservación postcosecha: un análisis de calidad de 44 introducciones y sus aplicaciones agroindustriales. Memorias IV Seminario Internacional de Agroforestería, Quibdó, Chocó, Colombia, Vol. 1, p 65. 2013.

42. Zhao G., Xin X, Chen W., Li X., Sun L., Sun L. Chemical constituents from Tithonia diversifolia and their chemotaxonomic significance. Biochemical Systematics and Ecology, 44: 250-254. 2012. 